\title{
$\begin{array}{lllllllll}\mathrm{I} & \mathrm{N} & \mathrm{S} & \mathrm{T} & \mathrm{I} & \mathrm{T} & \mathrm{U} & \mathrm{T} & \mathrm{E}\end{array}$
}

\section{Child Poverty High in Rural America}

WILLIAM P. O'HARE AND SARAH SAVAGE

$\mathrm{O}$ n Aug. 28, 2007, new data from the U.S. Census Bureau's American Community Survey show that 22 percent of rural children are living in poverty, up from 19 percent in 2000. On average, rates are highest in the nonmetropolitan South (27 percent) and have climbed the most in the nonmetropolitan Midwest (by 3.9 percentage points).

The child poverty rate is the most widely used indicator of child well-being because poverty is closely linked to undesirable outcomes in areas such as health, education, emotional welfare, and delinquency.

Table 1 shows child poverty rates for each state-and how child poverty rates have changed between 2000 and 2006 for the children living outside metropolitan areas in each state. (Data are collected in 2000 and 2006, but they actually reflect poverty in 1999 and 2005.) The data shown here are based on the official poverty measure as determined by the U.S. Office of Management and Budget. The official poverty measure consists of a series of income thresholds based on family size and composition. The 2006 poverty threshold was $\$ 20,444$ for a family of two adults and two children.

In 2003 the official definition of counties located in nonmetropolitan areas changed, which reduced the areas and population considered to be "rural." It is important to acknowledge this modification; however the change in definition has little impact on the poverty rates examined here.

\section{A U T H O R S}

Dr. William P. O’Hare (bbohare@comcast.net) is a Rural Fellow at the Carsey Institute and a Senior Fellow with the KIDS COUNT project at the Annie E. Casey Foundation.

Sarah Savage (ssavage@cisunix.unh.edu) is an Evaluation Fellow at the Carsey Institute and a Ph.D. student in sociology at UNH.

This fact sheet is available online at

http://carseyinstitute.unh.edu/FS_ruralpoverty_07.htm.

\section{Key results include:}

- The rural child poverty rate in 2006 ranges from a low of 9.1 percent in Connecticut to a high of nearly 34.7 percent in Mississippi.

- Rural child poverty rates increased between 2000 and 2006 in 37 of the 47 states where rural data was available (data were unavailable in 3 states and the District of Columbia).

- In 19 states, the increase in the rural child poverty rate was higher than the increase in the overall U.S. child poverty rate ( 3.0 percent) between 2000 and 2006.

- The state with the biggest percentage point increase in rural child poverty between 2000 and 2006 was Ohio (+6.8 percentage points), followed by Indiana ( +6.4 percentage points), and Maine ( +5.5 percentage points).

- Ten states showed a decrease in the rural child poverty rate, led by Maryland with a 4.0 percentage point decrease.

- Five states (Ohio, Indiana, Maine, Tennessee, and South Carolina) experienced increases of 5 percentage points or more in rural child poverty between 2000 and 2006.

- Three states (Mississippi, Louisiana, and New Mexico) all had rural child poverty rates above 30 percent in 2006, which reflects the pervasive child poverty problem in the rural South as well as areas with high percentages of minority populations.

\section{CARSEY I N S T I T U T E}

Building KNOWLEDGE FOR FAmilies AND Communities IN THE 21ST CENTURY
Huddleston Hall, 73 Main Street, Durham, NH 03824

(6o3) 862-2821 • www.carseyinstitute.unh.edu

The Carsey Institute Reports on Rural America are supported by the Annie E. Casey Foundation's initiative to strengthen rural families, the Ford Foundation, and the W.K. Kellogg Foundation. 
Table 1. Child Poverty in Nonmetropolitan AReAs by STATE: 2000 AND $2006^{1}$

\begin{tabular}{|c|c|c|c|c|c|c|c|}
\hline & & 2000 Census & & $2006 \mathrm{Ar}$ & erican Communi & ty Survey & \\
\hline & $\begin{array}{c}\text { Population under } \\
\text { age } 18^{*}\end{array}$ & $\begin{array}{c}\text { Population under } \\
\text { age } 18 \\
\text { below poverty }\end{array}$ & $\begin{array}{l}\text { Percent of population } \\
\text { under age } 18 \\
\text { below poverty }\end{array}$ & $\begin{array}{c}\text { Population under } \\
\text { age } 18^{*}\end{array}$ & $\begin{array}{l}\text { Population under } \\
\text { age } 18 \\
\text { below poverty }\end{array}$ & $\begin{array}{l}\text { Percent of population } \\
\text { under age } 18 \\
\text { below poverty }\end{array}$ & $\begin{array}{c}\text { Percentage point } \\
\text { change in poverty rate } \\
\text { from } 2000 \text { to } 2006\end{array}$ \\
\hline U.S. & $13,742,721$ & $2,644,052$ & 19.2 & $11,438,087$ & $2,539,740$ & 22.2 & 3.0 \\
\hline Alabama & 332,749 & 87,110 & 26.2 & 315,767 & 79,674 & 25.2 & -1.0 \\
\hline Alaska & 112,840 & 15,180 & 13.5 & 58,151 & 10,623 & 18.3 & 4.8 \\
\hline Arizona & 167,417 & 49,343 & 29.5 & 170,475 & 49,049 & 28.8 & -0.7 \\
\hline Arkansas & 331,938 & 81,708 & 24.6 & 268,053 & 76,256 & 28.4 & 3.8 \\
\hline California & 282,892 & 62,521 & 22.1 & 171,300 & 31,532 & 18.4 & -3.7 \\
\hline Colorado & 167,164 & 24,106 & 14.4 & 145,922 & 26,889 & 18.4 & 4.0 \\
\hline Connecticut & 34,966 & 2,336 & 6.7 & 65,900 & 5,993 & 9.1 & 2.4 \\
\hline Delaware & 34,417 & 5,273 & 15.3 & 36,929 & 7,269 & 19.7 & 4.4 \\
\hline District of Columbia & $\mathrm{N} / \mathrm{A}$ & $\mathrm{N} / \mathrm{A}$ & $\mathrm{N} / \mathrm{A}$ & $\mathrm{N} / \mathrm{A}$ & $\mathrm{N} / \mathrm{A}$ & N/A & $\mathrm{N} / \mathrm{A}$ \\
\hline Florida & 241,825 & 55,002 & 22.7 & 223,780 & 52,371 & 23.4 & 0.7 \\
\hline Georgia & 651,551 & 150,267 & 23.1 & 431,248 & 116,169 & 26.9 & 3.8 \\
\hline Hawaii & 84,919 & 14,387 & 16.9 & 86,148 & 12,651 & 14.7 & -2.2 \\
\hline Idaho & 220,968 & 34,694 & 15.7 & 129,965 & 21,886 & 16.8 & 1.1 \\
\hline Illinois & 437,147 & 64,090 & 14.7 & 357,444 & 70,093 & 19.6 & 4.9 \\
\hline Indiana & 428,843 & 49,635 & 11.6 & 341,268 & 61,289 & 18.0 & 6.4 \\
\hline lowa & 391,775 & 43,773 & 11.2 & 302,541 & 38,949 & 12.9 & 1.7 \\
\hline Kansas & 297,659 & 42,243 & 14.2 & 238,686 & 42,697 & 17.9 & 3.7 \\
\hline Kentucky & 498,717 & 127,783 & 25.6 & 408,818 & 113,363 & 27.7 & 2.1 \\
\hline Louisiana & 296,626 & 92,517 & 31.2 & 295,332 & 101,521 & 34.4 & 3.2 \\
\hline Maine & 188,129 & 28,321 & 15.1 & 110,490 & 22,713 & 20.6 & 5.5 \\
\hline Maryland & 91,722 & 13,636 & 14.9 & 66,456 & 7,224 & 10.9 & -4.0 \\
\hline Massachusetts & 54,791 & 5,654 & 10.3 & $\mathrm{~N} / \mathrm{A}$ & $\mathrm{N} / \mathrm{A}$ & $\mathrm{N} / \mathrm{A}$ & $\mathrm{N} / \mathrm{A}$ \\
\hline Michigan & 426,474 & 57,527 & 13.5 & 400,706 & 73,266 & 18.3 & 4.8 \\
\hline Minnesota & 365,389 & 41,266 & 11.3 & 312,986 & 41,850 & 13.4 & 2.1 \\
\hline Mississippi & 491,699 & 150,376 & 30.6 & 421,691 & 146,446 & 34.7 & 4.1 \\
\hline Missouri & 437,843 & 88,848 & 20.3 & 359,316 & 80,212 & 22.3 & 2.0 \\
\hline Montana & 150,910 & 30,806 & 20.4 & 139,063 & 25,559 & 18.4 & -2.0 \\
\hline Nebraska & 209,175 & 28,292 & 13.5 & 179,565 & 29,568 & 16.5 & 3.0 \\
\hline Nevada & 65,920 & 8,101 & 12.3 & 61,564 & 6,803 & 11.1 & -1.2 \\
\hline New Hampshire & 115,543 & 10,126 & 8.8 & 99,757 & 9,627 & 9.7 & 0.9 \\
\hline New Jersey & N/A & N/A & N/A & N/A & $\mathrm{N} / \mathrm{A}$ & N/A & N/A \\
\hline New Mexico & 229,836 & 68,609 & 29.9 & 177,750 & 53,420 & 30.1 & 0.2 \\
\hline New York & 350,537 & 60,687 & 17.3 & 324,943 & 63,898 & 19.7 & 2.4 \\
\hline North Carolina & 620,149 & 128,003 & 20.6 & 625,675 & 157,381 & 25.2 & 4.6 \\
\hline North Dakota & 90,858 & 15,228 & 16.8 & 76,044 & 11,903 & 15.7 & -1.1 \\
\hline Ohio & 545,599 & 73,234 & 13.4 & 524,534 & 105,850 & 20.2 & 6.8 \\
\hline Oklahoma & 336,924 & 77,161 & 22.9 & 306,953 & 82,274 & 26.8 & 3.9 \\
\hline Oregon & 221,997 & 39,533 & 17.8 & 184,211 & 40,256 & 21.9 & 4.1 \\
\hline Pennsylvania & 433,571 & 65,103 & 15.0 & 412,921 & 72,294 & 17.5 & 2.5 \\
\hline Rhode Island & 13,897 & 1,668 & 12.0 & $\mathrm{~N} / \mathrm{A}$ & $\mathrm{N} / \mathrm{A}$ & $\mathrm{N} / \mathrm{A}$ & $\mathrm{N} / \mathrm{A}$ \\
\hline South Carolina & 304,110 & 72,489 & 23.8 & 250,107 & 72,881 & 29.1 & 5.3 \\
\hline South Dakota & 130,019 & 26,332 & 20.3 & 103,688 & 20,987 & 20.2 & -0.1 \\
\hline Tennessee & 426,258 & 83,870 & 19.7 & 366,279 & 92,079 & 25.1 & 5.4 \\
\hline Texas & 827,383 & 205,766 & 24.9 & 731,057 & 199,362 & 27.3 & 2.4 \\
\hline Utah & 170,771 & 22,891 & 13.4 & 85,087 & 12,562 & 14.8 & 1.4 \\
\hline Vermont & 105,163 & 13,244 & 12.6 & 86,112 & 11,139 & 12.9 & 0.3 \\
\hline Virginia & 334,054 & 56,893 & 17.0 & 221,537 & 42,472 & 19.2 & 2.2 \\
\hline Washington & 250,389 & 49,332 & 19.7 & 173,282 & 36,469 & 21.0 & 1.3 \\
\hline West Virginia & 227,053 & 62,977 & 27.7 & 168,863 & 49,293 & 29.2 & 1.5 \\
\hline Wisconsin & 423,362 & 43,238 & 10.2 & 333,284 & 44,538 & 13.4 & 3.2 \\
\hline Wyoming & 88,783 & 12,873 & 14.5 & 81,373 & 8,891 & 10.9 & -3.6 \\
\hline Midwest & $4,184,143$ & 573,706 & 13.7 & $3,530,062$ & 621,202 & 17.6 & 3.9 \\
\hline Northeast & $1,227,909$ & 179,817 & 14.6 & $1,105,189$ & 185,913 & 16.8 & 2.2 \\
\hline South & $6,047,175$ & $1,450,831$ & 24.0 & $5,138,545$ & $1,396,035$ & 27.2 & 3.2 \\
\hline West & $2,214,806$ & 432,376 & 19.5 & $1,664,291$ & 336,590 & 20.2 & 0.7 \\
\hline
\end{tabular}

$\mathrm{N} / \mathrm{A}=$ Not applicable

${ }^{1}$ Metropolitan areas are defined based on the the U.S. Census Bureau's definitions as of June 2003. The full list of U.S. counties and cities/ towns used to define metropolitan areas is available online at http://www.census.gov/population/estimates/metro-city/03mfips.txt.

${ }^{*}$ for whom poverty is determined 